\title{
Average Time Spent by Lévy Flights and Walks on an Interval with Absorbing Boundaries
}

\author{
S. V. Buldyrev ${ }^{1}$, S. Havlin ${ }^{1,2}$, A. Ya. Kazakov ${ }^{3}$, M. G. E. da Luz ${ }^{4}$, E. P. Raposo ${ }^{5}$, \\ H. E. Stanley ${ }^{1}$, and G. M. Viswanathan ${ }^{1,6}$ \\ ${ }^{1}$ Center for Polymer Studies and Department of Physics, Boston University, Boston, MA 02215 \\ ${ }^{2}$ Gonda-Goldschmied Center and Department of Physics, Bar Ilan University, Ramat Gan, Israel \\ ${ }^{3}$ Mathematical Department, State University of Aerospace Instrumentation, 190000, Bol'shaya \\ Morskaya, 67, S.-Petersburg, Russia \\ ${ }^{4}$ Departamento de Física, Universidade Federal do Paraná, 81531-990, Curitiba-PR, Brazil \\ ${ }^{5}$ Laboratório de Física Teórica e Computacional, Departamento de Física, Universidade Federal \\ de Pernambuco, 50670-901, Recife-PE Brazil \\ ${ }^{6}$ Departamento de Física, Universidade Federal de Alagoas, 57072-970, Maceió-AL, Brazil
}

(EZ7284 19 May 2001)

\begin{abstract}
We consider a Lévy flyer of order $\alpha$ that starts from a point $x_{0}$ on an interval $[O, L]$ with absorbing boundaries. We find a closed-form expression for the average number of flights the flyer takes and the total length of the flights it travels before it is absorbed. These two quantities are equivalent to the mean first passage times for Lévy flights and Lévy walks, respectively. Using fractional differential equations with a Riesz kernel, we find exact analytical expressions for both quantities in the continuous limit. We show that numerical solutions for the discrete Lévy processes converge to the continuous approximations in all cases except the case of $\alpha \rightarrow 2$, and the cases of $x_{0} \rightarrow 0$ and $x_{0} \rightarrow L$. For $\alpha>2$, when the second moment of the flight length distribution exists, our result is replaced by known results of classical diffusion. We show that if $x_{0}$ is placed in the vicinity of absorbing boundaries, the average total length has a minimum at $\alpha=1$, corresponding to the Cauchy distribution. We discuss the relevance of this result to the problem of foraging, which has received recent attention in the statistical physics literature.
\end{abstract}

\section{INTRODUCTION}

In the past two decades, Lévy flights and Lévy walks (see [1-10]) found numerous applications in natural sciences. Realizations of Lévy flights in physical phenomena are very diverse, including fluid dynamics, dynamical systems, and statistical mechanics.

In general, Lévy flights and Lévy walks model anomalous diffusion, which is governed by rare but extremely large jumps of diffusing particles. Both Lévy walks and Lévy flights are characterized by broad distributions of their step lengths, for which the second moment does not exist. Lévy walks and Lévy flights of order $\alpha<2$ have distributions of step lengths 
with diverging moments of order $m \geq \alpha$ and converging moments of order $m<\alpha$. Hence, the classical central limit theorem, which governs the behavior of the Brownian motion, is not applicable. According to the generalized central limit theorem [4, 11], the probability density $\Psi(x, n)$ of the displacement $x$ of Lévy flights converges after many steps to the Lévy stable distribution of order $\alpha$ :

$$
\Psi(x, n)=\frac{1}{\pi} \int_{0}^{\infty} \exp \left(-n \ell_{0}^{\alpha} q^{\alpha}\right) \cos (q x) d q,
$$

where $\ell_{0}$ is the characteristic width of the distribution of a single step and $n$ is number of steps. This distribution is a generalization of the Gaussian distribution, and is characterized for asymptotically large displacements by the power law decay of its density with the exponent

$$
\mu=\alpha+1
$$

There are several definitions of Lévy walks and Lévy flights, which differ in terms of their spatial-temporal correlations (see e.g. [10]). Here we will restrict ourselves to the definition [7]. Accordingly, we assume that for Lévy flights the duration of each step is constant, so that velocity is proportional to the step length. Hence, the time of travel is proportional to the number of steps. Consequently, for Lévy flights, the mean-square displacement does not exist as a function of time. This property impedes direct applications of Lévy flights to physical phenomena.

In Lévy walks, walkers travel with constant velocity, which is independent of the step length. Hence, the time of travel is proportional to the total path length. Consequently, the mean-square displacement exists as function of time, but grows faster than linearly. This property makes Lévy walks applicable for modeling superdiffusion. However, the time evolution of Lévy flights is simpler than that of Lévy walks. Hence, in the following we will derive our results for Lévy flights, keeping in mind that the total path length of the Lévy flights corresponds to total time of travel in Lévy walks. In the continuous limit, Lévy flight process is described by the superdiffusion equation, which includes integer first order derivative with respect to time and fractional Riesz operator with respect to spatial coordinates [3, 1, 12 16. Here we will restrict our study to only this class of equations.

Note that usually anomalous diffusion is modeled by the equations of the SchneiderWyss type [17], which include fractional derivatives with respect to time and usual Laplace or Fokker-Planck operators with respect to spatial coordinates [3, 4,18 24]. In this case, the presence of absorbing boundaries can be treated the same way as in normal diffusion, since after the separation of variables, the solution can be expressed as series of the usual eigenfunctions of the boundary problem for the Laplace or Fokker-Planck operator [16,21,23].

In the absence of boundaries, the generalized central limit theorem allows us to treat Lévy flight diffusion with the help of fractional differential equations [3, 4, 6, 9, 9,12 [15]. In the presence of boundaries, the validity of fractional derivative formalism is less clear. Note that the problem of the discrete Lévy flights is finite, since it involves the characteristic width $\ell_{0}$ of the distribution of discrete steps. Thus the problem of Lévy flights in the finite domain of linear size $L$ must depend on the ratio $M \equiv L / \ell_{0}$. The transition from discrete Lévy flights to the fractional differential equation involves transition $\ell_{0} \rightarrow 0$. Consequently, the total number of flights and total path length diverge as powers of $M$. Since the same is true 
for the total path length of the Brownian walker, this problem can be solved by introducing fractional diffusion coefficient, the same way it is done in the usual diffusion equation. We will address this point in Section IV.

Lévy flights in a slab geometry with absorbing boundaries have been used to model the transmission of light through cloudy atmosphere [26]. Using heuristic arguments confirmed by numerical simulations, ref. [26] found the scaling behavior of the transmission probability of a photon through a slab of width $L$ and the total geometrical path length of transmitted and reflected light. This behavior was experimentally observed in [27. We analytically derive an exact expression for the transmission probability in Section IV.

Very recently [16], the approximate expressions for the mean first passage time for both Schneider-Wyss sub-diffusion equation [17] and superdiffusion equation [13] have been obtained by separation of variables. The latter case exactly corresponds to the Lévy flight problem, which we treat here (see Section IV). The problem of Lévy walks on a finite interval with absorbing boundaries has already been addressed in [25]. In that paper, the authors used an integral equation approach and performed the Laplace transform in the temporal domain. They found the asymptotic behavior of the survival probability, which is related to the asymptotic behavior of the first passage time. An alternative approach to the Lévy walk problem which employs the fractional Kramers equations can be found in [18]. However, as far as we know, exact expressions for the mean first passage time for Lévy flights and Lévy walks have not been derived.

The structure of the paper is the following. In Sections II and III, we find the mean time of travel before absorption for both discrete Lévy flights and Lévy walks as solutions of Fredholm integral equations of the second kind [28] with a power-law kernel truncated by a cut-off at small distance $\ell_{0}$.

In Section IV, we treat these equations in the limit $\ell_{0} \rightarrow 0$ and reduce our problem to the solution of fractional differential equations with Riesz kernels [3, 12, 24, 29,. These equations were previously applied in the plane contact problem [30] of linear creep theory and were solved using spectral relationships with Jacoby polynomials [24,31] and by the Sonin inversion formula [31,32]. We also show how our method is related to the method of separation of variables first applied to the partial fractional differential equation of superdiffusion in [16.

In Section $\mathrm{V}$, we compare our analytical solutions obtained in the continuous limit $\ell_{0} \rightarrow 0$ with the numerical solutions of the Fredholm equations obtained for discrete Lévy flights. We show that fractional differential equations can serve as good approximations for Lévy flights with absorbing boundaries for $\alpha<2$. We also show that these approximations break down when $\alpha \rightarrow 2$ and in the vicinity of the absorbing boundaries.

Finally, in Section VI, we discuss the relevance of our results to the problem of biological foraging. Recently, Lévy flights have been used to model animal foraging [7],33 37] and cell diffusion [38]. It has been suggested [36] that Lévy flights with $\alpha=1$ provide the optimal strategy of foraging in case of sparse food sites, if any food site can be revisited. This suggestion was based on the optimization of foraging efficiency, defined to be the inverse of the average total path length of the flyer before the flyer is absorbed by traps randomly distributed with certain density in $d$-dimensional space. The average total path length has been approximated [36] as a product of the average length of a single flight and the average number of flights before the flyer is absorbed by traps. It has been shown [36] that this 
product has a maximum at $\alpha=1$, if the starting point of the flyer $x_{0}$ is selected in the vicinity of the absorbing boundary. Here we confirm this result in the one-dimensional case using both an analytical expression for the average total length of flights obtained in the continuous limit and the numerical solution for the discrete Lévy process. We show that for the case of $x_{0}$ in the vicinity of the absorbing boundary, discrete and continuous solutions have the same power law asymptotic behavior, but their amplitudes are different. As a consequence, the continuous limit approximation has an additional minimum at $\alpha \rightarrow 2$, which is absent in the discrete case. This finding indicates that the fractional differential equation approach to Lévy flights breaks down in the vicinity of the absorbing boundary.

In Appendix A, we derive the fractional differential operator for the Lévy flight problem with absorbing boundaries. In Appendix B, we derive exact analytical expressions for the number of flights and the total length traveled before absorption, using the Sonin inversion formula for the Riesz fractional equation.

\section{MEAN NUMBER OF FLIGHTS}

Consider a Lévy flight that starts at point $x_{0}$ of the interval $[0, L]$ with absorbing boundaries. The flyer makes independent subsequent flights of variable random lengths $\ell$ with equal probability in both directions. The length of each flight is taken from the power law distribution

$$
P(|\ell|>r)=\left(\ell_{0} / r\right)^{\alpha}
$$

where the exponent $\alpha$ can vary between 0 and 2 , and $\ell_{0}$ is the minimal flight length, which serves as lower cutoff of the distribution. The probability density of the flight length is given by

$$
p(\ell)=\frac{\alpha \ell_{0}^{\alpha}}{2} \frac{\theta\left(|\ell|-\ell_{0}\right)}{|\ell|^{\alpha+1}},
$$

where $\alpha \ell_{0}^{\alpha} / 2$ is a normalization constant and $\theta(x)=1$ for $x>0$ or 0 otherwise. The exponent $\mu$ of refs. 35, 36 is identical to $\alpha+1$. When $\alpha>2$, the second moment of the flight distribution converges and the process becomes equivalent to normal diffusion. As soon as the flyer lands outside the interval $[0, L]$, the process is terminated. Instead of the probability density Eq. (44) one could use any power-law decaying density [4,25] regularized at small distances $\ell_{0}$, including Lévy stable distribution Eq. (11) with $n=\pi /[2 \Gamma(\alpha) \sin (\pi \alpha / 2)]$, where $\Gamma(\alpha) \equiv \int_{0}^{\infty} t^{\alpha-1} e^{-t} d t$ is Euler $\Gamma$-function [39]. This value of $n$ is selected so that the asymptotic behavior of Eq. (四) coincides with Eq. (4) [4]. We use a truncated power-law density for the simplicity of analytical treatment.

We are interested in two quantities: the average number of flights before absorption $\langle n\rangle$ and the average total distance $\langle S\rangle$ traveled before absorption. Note that we consider the length of the last flight to be equal to the distance from the previous landing point to the boundary of the interval which flyer crosses during its last flight. This condition makes our problem equivalent to the problem of Lévy walks [25] with the time defined to be equal to the sum of the flight lengths. 
Suppose that the probability density of finding a Lévy flyer at point $x$ after $n$ flights is $P_{n}(x)$. Then the probability density after $n+1$ flights is given by the convolution of the probability density $P_{n}(x)$ and the probability density of the next flight $p(x)$ given by Eq. (田)

$$
P_{n+1}(y)=\int_{0}^{L} p(y-x) P_{n}(x) d x
$$

Let $\mathcal{L}_{\alpha}\left(\ell_{0}\right)$ be an integral operator with kernel $p(x-y)$ which is defined on a function $f(x)$ of an interval $[O, L]$ as

$$
\left[\mathcal{L}_{\alpha} f\right](y) \equiv \frac{\alpha \ell_{0}^{\alpha}}{2} \int_{0}^{L} \frac{f(x) \theta\left(|y-x|-\ell_{0}\right) d x}{|y-x|^{\alpha+1}} .
$$

One can see, that $\mathcal{L}_{\alpha}$ is a self-conjugate operator with respect to a scalar product $(f, g)=$ $\int_{0}^{L} f(x) g(x) d x$. It can be shown that for any continuous function $f, \int_{0}^{L}\left|\mathcal{L}_{\alpha} f\right| d x \leq[1-$ $\left.(2 / M)^{\alpha}\right] \int_{0}^{L}|f| d x$, where $M=L / \ell_{0}$. The value $\left[1-(2 / M)^{\alpha}\right]$ can be regarded as the norm of the operator $\mathcal{L}_{\alpha}$. It has a physical meaning of the survival probability, i.e. the probability for the flight that starts at the center of the interval to stay unabsorbed, which is less than one.

The distribution after $n$ flights is given by

$$
P_{n}(x)=\left[\mathcal{L}_{\alpha}^{n} P_{0}\right](x) .
$$

The initial probability density of the flyer located at position $x_{0}$ is the Dirac delta function, $P_{0}(x)=\delta\left(x-x_{0}\right)$. The probability that the flyer remains unabsorbed after $n$ flights,

$$
\int_{0}^{L}\left[\mathcal{L}_{\alpha}^{n} P_{0}\right](x) d x<\left[1-(2 / M)^{\alpha}\right]^{n}
$$

decays exponentially with $n$. The probability that the flyer is absorbed exactly on the $n^{\text {th }}$ flight is

$$
P_{n}=\int_{0}^{L}\left[\left(\mathcal{L}_{\alpha}^{n-1}-\mathcal{L}_{\alpha}^{n}\right) P_{0}\right](x) d x
$$

and thus, the average number of flights spent by the flyer on the interval is

$$
\langle n\rangle=\sum_{n=1}^{\infty} P_{n} n=\int_{0}^{L} \sum_{n=0}^{\infty}\left[\mathcal{L}_{\alpha}^{n} P_{0}\right](x) d x=-\int_{0}^{L}\left[\left(\mathcal{L}_{\alpha}-\mathcal{I}\right)^{-1} P_{0}\right](x) d x
$$

The infinite sum in Eq. (10) converges, since the norm of $\mathcal{L}_{\alpha}$ is less than one. Here $\left(\mathcal{L}_{\alpha}-\mathcal{I}\right)^{-1}$ is the inverse operator with respect to the operator $\mathcal{L}_{\alpha}-\mathcal{I}$, and $\mathcal{I}$ is the unity operator. Since the operator $\left(\mathcal{L}_{\alpha}-\mathcal{I}\right)^{-1}$ is also a self-conjugate operator, and $P_{0}(x)=\delta\left(x-x_{0}\right)$, we have

$$
\langle n\rangle=\left[\left(\mathcal{L}_{\alpha}-\mathcal{I}\right)^{-1} h\right]\left(x_{0}\right)
$$

where $h(x)=-1$ is the constant function. This equation can be rewritten as a Fredholm integral equation of the second kind [28] with the kernel $p\left(x_{0}-x_{1}\right)$ : 


$$
\left\langle n\left(x_{0}\right)\right\rangle=1+\int_{0}^{L}\left\langle n\left(x_{1}\right)\right\rangle p\left(x_{0}-x_{1}\right) d x_{1} .
$$

Equation (12) can be interpreted as a recursive method to determine $\left\langle n\left(x_{0}\right)\right\rangle$. Indeed, the average total number of flights for the process that start at $x_{0}$ is equal to one (contribution from the very first flight, which always takes place) plus the convolution of the average total number of flights for the processes that start from all possible landing points $x_{1}$ of the first flight inside the interval and the probability density $p\left(x_{0}-x_{1}\right)$ to land at these points after the first flight.

In general, consider a quantity $Q\left(x_{0}\right)=\left\langle\sum_{i=1}^{\infty} q_{i}\right\rangle$, where $q_{i}=q\left(x_{i-1}, x_{i}\right)$ is a function of the starting point $x_{i-1}$ and ending point $x_{i}$ of the $i$-th flight, and \langle\rangle denotes the average over all possible processes starting at point $x_{0}$. Then, in analogy with the average number of flights, such a quantity must satisfy a recursion relation

$$
Q\left(x_{0}\right)=\left\langle q_{0}\left(x_{0}\right)\right\rangle+\int_{0}^{L} Q\left(x_{1}\right) p\left(x_{0}-x_{1}\right) d x_{1}
$$

where $\left\langle q_{0}(x)\right\rangle \equiv \int_{-\infty}^{\infty} p\left(x_{1}-x_{0}\right) q\left(x_{0}, x_{1}\right) d x_{1}$. Note that if $x_{1}$ is outside the interval $[0, L]$, the particle is absorbed by one of the boundaries and the value $q\left(x_{0}, x_{1}\right)$ should be defined according to its physical meaning for the absorbed particle. Equation (13) is identical to Eq.(11) with $h(x)=-\left\langle q_{0}(x)\right\rangle$.

As an example of application of Eq. (13), let us consider quantity $Q\left(x_{0}\right)$ to be the total flux through the right boundary. This flux is related to the transmission probability of photons through the clouds [26]. By definition, the flux through the right boundary is equal to the probability $P_{r}\left(x_{0}\right)$ of the absorption of the flyer that starts at point $x_{0}$ by the absorbing boundary $x=L$. In this case, quantity $q$ must be defined as $q\left(x_{0}, x_{1}\right)=\theta\left(x_{1}-L\right)$. The very first flight is absorbed by the right boundary with probability $p_{r}\left(x_{0}\right) \equiv\left\langle q_{0}\left(x_{0}\right)\right\rangle=$ $\int_{L}^{\infty} p\left(x_{1}-x_{0}\right) d x_{1}$, or after integration

$$
p_{r}\left(x_{0}\right)=\left\{\begin{array}{cl}
{\left[\ell_{0} /\left(L-x_{0}\right)\right]^{\alpha} / 2,} & 0 \leq x_{0}<L-\ell_{0} \\
1 / 2, & L-\ell_{0} \leq x_{0} \leq L
\end{array} .\right.
$$

Therefore, $P_{r}\left(x_{0}\right)$ satisfies Eq. (13) with $\left\langle q_{0}\left(x_{0}\right)\right\rangle=p_{r}\left(x_{0}\right)$ and Eq. (11) with $h\left(x_{0}\right)=$ $-p_{r}\left(x_{0}\right)$. In the next section, we will apply this method to define the total path length of the flyer.

\section{AVERAGE TOTAL PATH LENGTH TRAVELED BY THE FLYER}

The average total path length traveled by a Lévy flyer before absorption is equivalent to the total time spent by a Lévy walker before absorption [25]. The evolution of the probability density of Lévy walks was studied [25] in terms of time. This approach leads to integral equations involving integration over time and space. Here we restrict our study to the problem of the average total path length before absorption of discrete Lévy flights. This particular problem can be solved in much simpler terms.

In the absence of the absorbing boundaries, the average flight length with probability density $p(\ell)$ of Eq. (4) is given by $\langle|\ell|\rangle=\int_{-\infty}^{+\infty}|\ell| p(\ell) d \ell$, which is independent of the starting point and diverges for $\alpha \leq 1$. In the presence of the absorbing boundaries, the flight starting 
from a point $y$ cannot exceed the distances $y$ and $L-y$ from this point to the boundaries. One can show that the average length of a flight that starts from a point $y$ of an interval $\left[\ell_{0}, L-\ell_{0}\right]$ is given by

$$
\begin{aligned}
s(y) \equiv & \langle|\ell(y)|\rangle=\frac{\alpha \ell_{0}^{\alpha}}{2}\left[\int_{0}^{y-\ell_{0}} \frac{d x}{(y-x)^{\alpha}}+\int_{y+\ell_{0}}^{L} \frac{d x}{(x-y)^{\alpha}}\right. \\
& \left.+y \int_{-\infty}^{0} \frac{d x}{(y-x)^{\alpha+1}}+(L-y) \int_{L}^{\infty} \frac{d x}{(x-y)^{\alpha+1}}\right],
\end{aligned}
$$

which converges for $\alpha>0$. If $\alpha \neq 1$, Eq. (15) reduces to

$$
s(y)=\frac{\ell_{0}}{2(1-\alpha)}\left[\left(\frac{\ell_{0}}{y}\right)^{\alpha-1}+\left(\frac{\ell_{0}}{L-y}\right)^{\alpha-1}-2 \alpha\right] .
$$

In case $\alpha=1$, we have from Eq. (15)

$$
s(y)=\frac{\ell_{0}}{2}\left[\ln \left(\frac{y}{\ell_{0}}\right)+\ln \left(\frac{L-y}{\ell_{0}}\right)+2\right] .
$$

If $0 \leq y<\ell_{0}$, Eq. (16) must be replaced by

$$
s(y)=\frac{y}{2}+\frac{\ell_{0}}{2(1-\alpha)}\left[\left(\frac{\ell_{0}}{L-y}\right)^{\alpha-1}-\alpha\right],
$$

or by

$$
s(y)=\frac{L-y}{2}+\frac{\ell_{0}}{2(1-\alpha)}\left[\left(\frac{\ell_{0}}{y}\right)^{\alpha-1}-\alpha\right],
$$

if $L-\ell_{0}<y \leq L$. Analogous changes must be made in Eq. (17).

Thus, according to Eq. (13), the total average path length for the process that starts at point $x_{0}$ is

$$
\left\langle S\left(x_{0}\right)\right\rangle=s\left(x_{0}\right)+\int_{0}^{L}\left\langle S\left(x_{1}\right)\right\rangle p\left(x_{0}-x_{1}\right) d x_{1}
$$

or

$$
\left\langle S\left(x_{0}\right)\right\rangle=-\left[\left(\mathcal{L}_{\alpha}-\mathcal{I}\right)^{-1} s\right]\left(x_{0}\right) .
$$

This equation is identical to the Eq. (11) in which function $h(x)=-s(x)$. We will solve Eqs. (12) and (20) numerically in Section V. 


\section{THE CONTINUOUS LIMIT}

Appendix A shows that for $\alpha<2$, operator $\mathcal{L}_{\alpha}\left(\ell_{0}\right)-\mathcal{I}$ tends to zero when $\ell_{0} \rightarrow 0$ for any function $f(x)$ that has second derivative $f^{\prime \prime}(x)$ for $0<x<L$ and has finite limits $f(0)$ and $f(L)$. It can also be shown that for such functions and $\alpha<2$ there exists an operator 3.,24, 42

$$
\mathcal{D}_{\alpha} \equiv \lim _{\ell_{0} \rightarrow 0} \ell_{0}^{-\alpha}\left[\mathcal{L}_{\alpha}\left(\ell_{0}\right)-\mathcal{I}\right]
$$

The result of this operator acting on any such function is defined as

$$
\left[\mathcal{D}_{\alpha} f\right](y)=\mathrm{V} . \mathrm{P} . \int_{0}^{L} \frac{\operatorname{sgn}(x-y) f^{\prime}(x) d x}{2|y-x|^{\alpha}}-\frac{f(0)}{2 y^{\alpha}}-\frac{f(L)}{2(L-y)^{\alpha}},
$$

where $f^{\prime}(x)$ is the first derivative of the function $f(x)$. This operator is a self-conjugate operator similar to the double differentiation operator $d^{2} / d x^{2}$. It can be expressed [3,24] as the linear combination of right and left Riemann-Liouville fractional derivatives of the order $\alpha$. The difference of the two operators

$$
d_{\alpha}\left(\ell_{0}\right) \equiv \ell_{0}^{-\alpha}\left[\mathcal{L}_{\alpha}\left(\ell_{0}\right)-\mathcal{I}\right]-\mathcal{D}_{\alpha}
$$

decays as $\ell_{0}^{2-\alpha}$ when $\ell_{0} \rightarrow 0$. Appendix A also shows that the leading term of the operator $d_{\alpha}$ is proportional to the operator of the second derivative

$$
d_{\alpha}\left(\ell_{0}\right)=\ell_{0}^{2-\alpha} \frac{\alpha}{2(\alpha-2)} \frac{d^{2}}{d x^{2}}+o\left(\ell_{o}^{2-\alpha}\right) .
$$

In analogy with the diffusion equation with continuous time, we can define a superdiffusion equation [3, 12, 13, 16] based on Lévy flights. Instead of the discrete process defined by Eq. (5), one can write

$$
\frac{\partial P(x, t)}{\partial t}=\frac{\ell_{0}^{\alpha}}{t_{0}} \mathcal{D}_{\alpha} P(x, t)
$$

where $t_{0}$ is the duration of each flight, and $\ell_{0}^{\alpha} / t_{0}$ is the fractional analog of the diffusion coefficient. Note that $\ell_{0}$ plays a role similar to the mean free path, and $t_{0}$ plays the role of the mean collision interval.

The operator $\mathcal{D}_{\alpha}$ has an orthogonal normalized set of eigenfunctions $f_{k}(x)$, such that $\mathcal{D}_{\alpha} f_{k}(x)=\lambda_{k} f_{k}(x)$, and $f_{k}(0)=f_{k}(L)=0$ 29,40. Similarly to the solution of usual diffusion equation, the solution of Eq. (26) can be expressed via separation of variables as a series of eigenfunctions

$$
P(x, t)=\sum_{k=1}^{\infty} e^{\lambda_{k} \ell_{0}^{\alpha} t / t_{0}} f_{k}(x) \int_{0}^{L} f_{k}(y) P(y, 0) d y .
$$

In Ref. [16], where the method of separation of variables for the superdiffusion equation on a finite interval has been first proposed, it has been assumed that the eigenvalues $\lambda_{k}$ asymptotically behave at large $k$ as $\lambda_{k} \sim-(k / L)^{\alpha}<0$ and that the eigenfunctions $f_{k}(x)$ 
can be well approximated by the eigenfunctions $\sqrt{2 / L} \sin (x \pi k / L)$ of the Laplace operator with absorbing boundary conditions. Numerical studies 41] confirm these assumptions but show that eigenfunctions $f_{k}$ and sines have different behavior near absorbing boundaries, namely, $f_{k}(x) \sim x^{\alpha / 2}$ as $x \rightarrow 0$.

Having defined the properties of the operator $\mathcal{D}_{\alpha}$, we can derive the closed form expression for the average time spent by the continuous Lévy flight process on the interval. Formal substitution of Eq. (22) into Eq. (11) yields

$$
\langle t\rangle=t_{0}\langle n\rangle=\frac{t_{0}}{\ell_{0}^{\alpha}}\left[\mathcal{D}_{\alpha}^{-1} h\right]\left(x_{0}\right)=\frac{t_{0}}{\ell_{0}^{\alpha}} g\left(x_{0}\right),
$$

where function $g(x)$ satisfies the equation

$$
\mathcal{D}_{\alpha} g(x)=h(x)=-1 \text {. }
$$

Note that $g(x)$ has to satisfy boundary conditions $g(0)=0, g(L)=0$. Otherwise, according to Eq. (23), the right hand side of Eq. (29) would contain singularities. In the general case, the equation

$$
\text { V.P. } \int_{0}^{L} \frac{\operatorname{sgn}(y-x) f^{\prime}(y) d y}{2|y-x|^{\alpha}}=h(x)
$$

with absorbing boundary conditions

$$
f(0)=f(L)=0
$$

belongs to a known class of generalized Abel integral equations with Riesz fractional kernel 24, 29 31. It can be shown 29,31 that such an equation with boundary conditions (31) has a unique solution which can be obtained via spectral relationships for Jacobi polynomials 24, 31] or by the Sonin inversion formula 31,32 (see Appendix B). Similar inversion formulae are given in ref. [29]. In the case $h(x)=-1$, the solution can be expressed in elementary functions:

$$
g(x)=\frac{2 \sin (\pi \alpha / 2)}{\pi \alpha}[(L-x) x]^{\alpha / 2} .
$$

One can verify this solution by performing contour integration around the cut $[0, L]$ on the complex plane and computing the residue of the integrand at infinity. It should be pointed out that $g(x)$ can be expanded in a series of eigenfunctions $f_{k}(x)$ :

$$
g\left(x_{0}\right)=-\sum_{k=1}^{\infty} \lambda_{k}^{-1} f_{k}\left(x_{0}\right) \int_{0}^{L} f_{k}(x) d x
$$

This expansion is similar in spirit to an approximation, obtained in [16], where the exact eigenfunctions were approximated by sines. Although approximation 16 correctly predicts the power law dependence $g\left(x_{0}\right) \sim L^{\alpha}$ for the points $x_{0}$ in the center of the interval, it differs from Eq. (32) in the proportionality coefficient and in the behavior near absorbing boundaries 41]. 
Note that for $\alpha>2$, Eq. (26) should be replaced [42] by the standard diffusion equation with diffusion coefficient $D=\ell_{o}^{2} \alpha /\left[t_{o} 2(\alpha-2)\right]$. In this case, the average time spent by the flyer before absorption is given by the classical equation $\langle t\rangle=x(L-x) /(2 D)$. Note that the diffusion coefficient $D$ resembles the proportionality coefficient in Eq. (25).

One can argue that the expression (32) may yield the average number of flights taken by the discrete Lévy flight process in the limit of $\ell_{0} \rightarrow 0$. Indeed, according to Eq. (24)

$$
\mathcal{L}_{\alpha}\left(\ell_{0}\right)-\mathcal{I}=\ell_{0}^{\alpha}\left[\mathcal{D}_{\alpha}+d_{\alpha}\left(\ell_{0}\right)\right],
$$

where operator $d_{\alpha}\left(\ell_{0}\right) \rightarrow 0$, as $\ell_{0} \rightarrow 0$. Formally expanding $\left(\mathcal{L}_{\alpha}-\mathcal{I}\right)^{-1}$ in powers of $d_{\alpha}$, we obtain

$$
\left[\mathcal{L}_{\alpha}-\mathcal{I}\right]^{-1}=\ell_{0}^{-\alpha}\left(\mathcal{D}_{\alpha}^{-1}-\mathcal{D}_{\alpha}^{-1} d_{\alpha} \mathcal{D}_{\alpha}^{-1}+\ldots\right)
$$

and thus

$$
\langle n\rangle=\ell_{0}^{-\alpha}\left\{g\left(x_{0}\right)-\left[\mathcal{D}_{\alpha}^{-1} d_{\alpha} g\right]\left(x_{0}\right)+\ldots\right\}
$$

Note that expansion (35) is formal and may not converge. We will test this assumption numerically in Section V. In order to distinguish the average number of flights for the discrete process, $\langle n\rangle$, from the continuous limit approximation, we will denote the latter by $n_{\alpha}(x)$ :

$$
n_{\alpha}\left(x_{0}\right) \equiv \ell^{-\alpha} g\left(x_{0}\right)=\frac{\sin (\pi \alpha / 2)}{\pi \alpha / 2}\left[\frac{\left(L-x_{0}\right) x_{0}}{\ell_{0}^{2}}\right]^{\alpha / 2}=\frac{\sin (\pi \alpha / 2) M^{\alpha}\left(z-z^{2}\right)^{\alpha / 2}}{\pi \alpha / 2},
$$

where $z \equiv x_{0} / L, M \equiv L / \ell_{0}$.

Analogously, we will denote the continuous limit approximation for the average total path length by $S_{\alpha}$. In the continuous limit, Eq. (21) should be replaced by Eq. (30) with $f(y)=S_{\alpha}(y), h(x)=\ell_{0}^{-\alpha} s(x)$, and absorbing boundary conditions (31). In this case (See Appendix B), Sonin formula leads to an expression containing hypergeometric functions:

$$
S_{\alpha}\left(x_{0}\right)=\frac{L(2-\alpha)}{2(1-\alpha)}\left[1-4 \frac{\psi_{\alpha}(z)+\psi_{\alpha}(1-z)}{\alpha(\alpha+2) \mathrm{B}\left(\frac{\alpha}{2}, \frac{\alpha}{2}\right)}\right]+\frac{2 L M^{\alpha-1} \sin \left(\frac{\pi \alpha}{2}\right)\left(z-z^{2}\right)^{\frac{\alpha}{2}}}{\pi(\alpha-1)},
$$

where $\mathrm{B}(a, b) \equiv \Gamma(a) \Gamma(b) / \Gamma(a+b)$ is Euler B-function,

$$
\psi_{\alpha}(z)=F\left(2-\frac{\alpha}{2}, \frac{\alpha}{2}, \frac{\alpha}{2}+2, z\right) z^{\frac{\alpha}{2}+1},
$$

and $F$ is the hypergeometric function [39]

$$
F(a, b, c, x) \equiv \frac{\Gamma(c)}{\Gamma(a) \Gamma(b)} \sum_{n=0}^{\infty} \frac{\Gamma(n+a) \Gamma(n+b) x^{n}}{\Gamma(n+1) \Gamma(n+c)} .
$$

In case $\alpha=1$, corresponding to the Cauchy distribution, the hypergeometric function $\psi_{\alpha}(z)$ can be expressed in elementary functions $\psi_{1}(z)=\frac{3}{4}\left[\frac{\pi}{2}+\sin ^{-1}(2 z-1)-2 \sqrt{z-z^{2}}\right]$ and Eq. (37) yields $S_{1}\left(x_{0}\right)=L \frac{2}{\pi} \sqrt{z-z^{2}} \ln M+O(1)$, where terms $O(1)$ do not depend on $M$ and can be found from Eq. (37) by L'Hôpital's rule. 
Note that the average total path length traveled before absorption by the Lévy flyer can be expressed in terms of survival probability $\Theta(t)$ of the Lévy walker: $\langle S\rangle=\int_{0}^{\infty} \Theta(t) d t$. According to [25], this survival probability exhibit for $t \rightarrow \infty$ asymptotic exponential decay $\Theta(t) \sim \exp \left(-\left|\Lambda_{1}\right| t\right)$, where $\Lambda_{1}$ is the main eigenvalue of the correspondent problem. Substituting $\Theta(t)$ by its asymptotic, we can estimate $\langle S\rangle \sim 1 /\left|\Lambda_{1}\right|$. Thus $\langle S\rangle$ and $1 /\left|\Lambda_{1}\right|$ must have identical asymptotic behavior for large $L$. Indeed, Eq. (37) yields $S_{\alpha}(x) \sim L$ for $0<\alpha<1$ and $S_{\alpha}(x) \sim L^{\alpha}$ for $1 \leq \alpha<2$, in complete agreement with asymptotic approximations of [25].

Finally, we will find the probability $P_{r}\left(x_{0}\right)$ of the absorption by the right boundary in the continuous limit. According to Eqs. (13) and (14), $P_{r}\left(x_{0}\right)$ should, in continuous case, satisfy Eq. (29) with $h\left(x_{0}\right)=-\ell_{0}^{-\alpha} p_{r}\left(x_{0}\right)$, i.e.

$$
\mathcal{D}_{\alpha} \mathcal{P}_{\nabla}\left(\S_{\prime}\right)=-\left(\mathcal{L}-\S_{\prime}\right)^{-\alpha} / \in
$$

For $x_{0}=0$, the flyer is immediately absorbed by the left boundary, so $P_{r}(0)=0$. For $x_{0}=L$, the flyer is immediately absorbed by the right boundary, so $P_{r}(L)=1$. Thus the second term in the expression (23) for $\mathcal{D}_{\alpha} \mathcal{P}_{\nabla}\left(\S_{\prime}\right)$ is equal to zero and the third term cancels out with the right hand side. Hence $P_{r}\left(x_{0}\right)$ satisfies homogeneous Eq. (30) and boundary conditions $P_{r}(0)=0, P_{r}(L)=1$. This solution can be expressed in terms of the homogeneous solution $\varphi_{0}(x)$ obtained in Appendix B:

$$
P_{r}\left(x_{0}\right)=\frac{\int_{0}^{x_{0}} \varphi_{0}(y) d y}{\int_{0}^{L} \varphi_{0}(y) d y}=\left(\frac{x_{0}}{L}\right)^{\frac{\alpha}{2}} \frac{F\left(\frac{\alpha}{2}, 1-\frac{\alpha}{2}, \frac{\alpha}{2}+1, \frac{x_{0}}{L}\right)}{\frac{\alpha}{2} B\left(\frac{\alpha}{2}, \frac{\alpha}{2}\right)},
$$

Note that the probability of the absorption by the left boundary, $P_{l}\left(x_{0}\right)=P_{r}\left(L-x_{0}\right)=$ $1-P_{r}\left(x_{0}\right)$. For $x_{0} \rightarrow 0$, the asymptotic behavior of $P_{r}\left(x_{0}\right)$ is given by $P_{r}\left(x_{0}\right) \sim\left(x_{0} / L\right)^{\alpha / 2}$ which is in complete agreement with the result of ref. [26] for the transmission probability of the photons through the clouds of depth $L$.

\section{NUMERICAL SOLUTION}

The goal of this section is to treat Eq. (12) and Eq. (20) numerically and to compare the results with the continuous limit solutions Eq. (36) and Eq. (37). To perform numerical integration of Eqs.(12) and (20), we replace the integration by summation and the kernel $p(x-y)$ by the matrix $A_{i j}, 0<i<M$, such that $A_{i i}=0$ and

$$
A_{i j}=\frac{1}{2}\left[\frac{1}{|i-j|^{\alpha}}-\frac{1}{(|i-j|+1)^{\alpha}}\right], \quad i \neq j .
$$

Accordingly, the average flight length $s(x)$ performed from the point $k=x / \ell_{0}$ is replaced by $M-1$-dimensional vector $\vec{s}$ with elements

$$
s_{k}=\frac{\ell_{0}}{2(1-\alpha)}\left[\frac{1}{k^{\alpha-1}}+\frac{1}{(M-k)^{\alpha-1}}-2 \alpha\right] .
$$

The average number of flights for the process that starts from point $k=x / \ell_{0}$ is 


$$
\langle n\rangle_{k}=\left(\sum_{m=0}^{\infty} A^{m} \overrightarrow{e_{k}} \cdot \vec{c}\right)=\left[(I-A)^{-1} \vec{c}\right]_{k},
$$

where $\vec{c}$ is the vector with all components equal to 1 , and $\overrightarrow{e_{k}}$ is a unit basis vector with components $e_{k i}=\delta_{k i}$, where $\delta_{k i}$ is the Kronecker delta. Analogously, average total length is equal to

$$
\langle S\rangle_{k}=\ell_{0}\left(\sum_{m=0}^{\infty} A^{m} \overrightarrow{e_{k}} \cdot \vec{s}\right)=\left[(I-A)^{-1} \vec{s}\right]_{k}
$$

The symmetric matrix $R=(A-I)^{-1}$ is the analog of the self-conjugate operator $(\mathcal{L}-\mathcal{I})^{-1}$. Using iterative techniques for matrix inversion, we obtain the numerical solutions for $\langle n\rangle$ and $\langle S\rangle$.

In Figure 1, we compare the numerical solution (42) for $\left\langle n\left(x_{0}\right)\right\rangle$ and the continuous limit approximation $n_{\alpha}\left(x_{0}\right)$ given by Eq. (36) for the case $x_{0}=L / 2$. In order to test the asymptotic convergence, we have to divide both functions by $(M / 2)^{\alpha}$. It can be seen that for $x_{0} / \ell_{0} \gg 1$, Eq. (36) provides good approximation for the average number of flights of the discrete process defined by Eq. (11). Studying the difference between the numerical values of $\langle n\rangle(M / 2)^{-\alpha}$ and the continuous approximation $2 \sin (\pi \alpha / 2) /(\pi \alpha)$ for $x_{0}=L / 2$, we confirm that for $2>\alpha>1$ this difference decays as $M^{\alpha-2}$ with $M \rightarrow \infty$. This is in agreement with Eq. (25). However, for $\alpha<1$ the difference between the numerical solution and the continuous approximation converges as $M^{-1}>M^{\alpha-2}$. The term $M^{-1}$ is proportional to the error of replacement of integration in Eq. (12) by summation.

In Fig. 2, we compare the numerical solution (43) for $\left\langle S\left(x_{0}\right)\right\rangle$ and the continuous limit approximation $S_{\alpha}\left(x_{0}\right)$ given by Eq. (37) for the case $x_{0}=L / 2$. In order to test the asymptotic convergence in this case, we have to divide both functions by $\left(L M^{\alpha-1}-L\right) /(\alpha-$ 1). It can be seen that for $x_{0} / \ell_{0} \gg 1$, Eq. (37) provides good approximation for the average total traveled length in the discrete process defined by Eq. (21)

Now we will examine the quality of the continuous limit approximation in the vicinity of the absorbing boundary. For simplicity we will study only the behavior of the average number of flights. The approximation for the total path length has similar problems. Figure 3 shows that for $x_{0} / \ell_{0}=1$, the correction terms in Eq. (35) cannot be neglected. As shown in Appendix A, the operator $d_{\alpha} \rightarrow 0$ for any fixed $x_{0}$ if $\ell_{0} \rightarrow 0$, but does not vanish if $x_{0}$ and $\ell_{0}$ both approach zero, so that their ratio $x / \ell_{0} \rightarrow r>0$. Accordingly, the value of $\langle n\rangle$ behaves for $x_{0} / \ell_{0}=r, M \rightarrow \infty$ as $\chi(\alpha)(r M)^{\alpha / 2}$, where $\chi(\alpha)>2 \sin (\pi \alpha / 2) /(\pi \alpha)$ is some unknown function that can be estimated numerically (see Fig. 3). It is likely that $\chi(\alpha)$ remains positive as $\alpha \rightarrow 2$. The analytical determination of the function $\chi(\alpha)$ remains an unsolved problem. Nevertheless, continuous approximation correctly predicts the leading factor $M^{\alpha / 2}$ for the average number of flights started in the vicinity of the absorbing boundary.

In summary, comparison of the numerical solutions for the mean first passage time of Lévy flights and Lévy walks and the exact solutions of these problems in the continuous case suggests that fractional differential equation for superdiffusion Eq. (26) with absorbing boundary conditions provides good approximation for discrete Lévy flights on a finite interval with absorbing boundaries. However this approximation breaks down when $\alpha \rightarrow 2$ and in the vicinity of the absorbing boundaries. 


\section{ANALYSIS OF THE TOTAL PATH LENGTH: IMPLICATIONS FOR BIOLOGICAL FORAGING}

Recently, biological foraging has been modeled by Lévy flights [7, 33 [37]. The case of non-destructive foraging (defined in [36] as case in which "target sites" can be revisited not just once but many times) corresponds to $x_{0}=\ell_{0}$, i.e. the forager starts its next search from the previously-visited food site, located at the origin. The prey may reappear at this site. Accordingly, coming back to the origin may be profitable in terms of foraging efficiency, which is defined [36] as the inverse average total path length before finding next food site. With help of Monte-Carlo simulations, it has been shown [36] that, in case of non-destructive foraging, the foraging efficiency has maximum at $\alpha=1$.

We confirm this result, using numerical solution (43). Figure 4 shows a semi-logarithmic plot of $\langle S\rangle$ versus $\alpha$ for $x_{0}=\ell_{0}$ and various values of $M$. On can see that the minima, $\alpha_{\min }(M)$, shift towards $\alpha=1$ as $M \rightarrow \infty$. Heuristic approximations 36, 37 suggest that foraging efficiency has a maximum at $1-\epsilon$, where $\epsilon \sim(\ln M)^{-2}$. Consequently, the average total path length should have minimum at the same point. Figure 5 confirms this prediction for the numerical solution. It shows the graph of $\alpha_{\min }(M)$ versus $[\ln (M)]^{-2}$, which is almost a straight line with an intercept $\alpha_{\min }(\infty) \approx 1$.

In the following, we will prove this result using the continuous limit approximation (37). Accordingly, we will find the behavior of $S_{\alpha}\left(x_{0}\right)$ for the case when the starting point $x_{0}$ is selected in the vicinity of the absorbing boundary and show that in this case $S_{\alpha}\left(x_{0}\right)$ has a minimum at $\alpha \rightarrow 1$. In oder to do this, we will present solution (37) in a more convenient form, which allows to separate leading singularities at $z \rightarrow 0$. After some transformations involving hypergeometric functions [39], we can rewrite Eq. (37) as follows:

$$
\begin{gathered}
S_{\alpha}\left(x_{0}\right)=\frac{2 L\left(z-z^{2}\right)^{\frac{\alpha}{2}}}{\alpha-1}\left[\frac{M^{\alpha-1} \sin \left(\frac{\pi \alpha}{2}\right)}{\pi}-\frac{(1-z) f_{1}(\alpha, z)}{\alpha \mathrm{B}\left(\frac{\alpha}{2}, \frac{\alpha}{2}\right)}\right] \\
-\frac{L(2-\alpha)}{2(\alpha-1)}\left[1-f_{2}(\alpha, z)(1-z)^{\frac{\alpha}{2}+1}-\frac{4 f_{3}(\alpha, z) z^{\frac{\alpha}{2}+1}}{\alpha(\alpha+2) \mathrm{B}\left(\frac{\alpha}{2}, \frac{\alpha}{2}\right)}\right],
\end{gathered}
$$

where

$$
\begin{gathered}
f_{1}(\alpha, z)=F\left(\alpha, 2, \frac{\alpha}{2}+1, z\right), \\
f_{2}(\alpha, z)=F\left(2-\frac{\alpha}{2}, \frac{\alpha}{2}, 1-\frac{\alpha}{2}, z\right),
\end{gathered}
$$

and

$$
f_{3}(\alpha, z)=F\left(2-\frac{\alpha}{2}, \frac{\alpha}{2}, \frac{\alpha}{2}+2, z\right) .
$$

The first term in solution (44) decreases as $z^{\alpha / 2}$, when $z \rightarrow 0$, while the second part decreases as $z$. If we take the starting point $x_{0}=r \ell_{0}$, where $r$ is constant, then $z=r / M$, where $M$ is large number, and we can separate the leading (with respect to $M$ ) terms in solution (44): 


$$
S_{\alpha}\left(r \ell_{0}\right)=r^{\alpha / 2} \frac{L}{\alpha-1}\left[\eta(\alpha) M^{\frac{\alpha}{2}-1}-\zeta(\alpha) M^{-\frac{\alpha}{2}}\right]+L \cdot O\left(M^{-1}\right)
$$

where

$$
\eta(\alpha)=\frac{2 \sin (\pi \alpha / 2)}{\pi}, \quad \zeta(\alpha)=\frac{2}{\alpha \mathrm{B}\left(\frac{\alpha}{2}, \frac{\alpha}{2}\right)}
$$

The above approximation accurately follows the solutions of the discrete problem for $\alpha<1$, when the term $\zeta(\alpha) M^{-\alpha / 2}$ dominates, but strongly deviate from that for $\alpha>1$, when the term $\eta(\alpha) M^{\frac{\alpha}{2}-1}$ dominates (see Fig. 4). The reason for these deviations is the truncation of the non-leading terms in the Eq. (34).

In contrast with the discrete solution and Monte-Carlo simulations of [36], expression Eq. (45) has two minima: one at $\alpha=1+\varepsilon(M)$ and another at $\alpha=2$. We will show that $\varepsilon(M) \rightarrow 0$ as $M \rightarrow \infty$. Let us expand $\eta$ and $\zeta$ in powers of $\varepsilon$ :

$$
\eta(1+\varepsilon)=\eta_{0}+\eta_{1} \varepsilon+\ldots, \quad \zeta(1+\varepsilon)=\zeta_{0}+\zeta_{1} \varepsilon+\ldots
$$

Note that $\eta_{0}=\zeta_{0}=2 / \pi$, and hence the expression (45) does not have a singularity at $\alpha=1$. The location of the minimum can be found by differentiation of the expansion for $S_{1+\varepsilon}\left(r \ell_{0}\right)$ with respect to $\varepsilon$ and equating the leading terms of the order of $\ln M$ :

$$
\varepsilon=-\frac{6\left(\eta_{1}+\zeta_{1}\right)+3 \eta_{0} \ln r}{\eta_{0}(\ln M)^{2}}+o\left([\ln M]^{-2}\right)=\frac{6-12 \ln 2-3 \ln r}{(\ln M)^{2}}+o\left([\ln M]^{-2}\right) .
$$

Indeed, Eq. (48) shows that $\varepsilon(M) \rightarrow 0$ as $M \rightarrow \infty$ and $r$ stays constant.

This analysis holds for any continuous functions $\eta$ and $\zeta$, so long as $\eta(1)=\zeta(1)$ and, therefore, is likely to be valid in the discrete case, in which, functions $\eta(\alpha)=\eta_{d}(\alpha)$ and $\zeta(\alpha)=\zeta_{d}(\alpha)$ do not satisfy Eq. (46). Note that $\eta_{d}(\alpha)$ can be expressed in terms of function $\chi(\alpha)$ shown in Fig 3., namely $\eta_{d}(\alpha)=\alpha \chi(\alpha)$. Analysis of Fig 3. shows that $\chi(2)>0$. Consequently, the minimum at $\alpha=2$ does not exist in the discrete case.

\section{SUMMARY}

We have studied Lévy Flights in a finite interval with absorbing boundaries. In Section II, we have derived expressions Eqs. (11) and (12) for the average total number of flights (mean first passage time). We also obtain a general recursion relation Eq. (13) for the average of the sum of arbitrary contributions from each flight in the form of the Fredholm integral equation of the second kind. We applied this method to derive the probability of absorption by one of the boundaries. In section III, we have derived expressions Eqs. (20) and (21) for the average total path length of the Lévy flights which is equivalent to the mean first passage time of the Lévy walks.

In Section IV, we have shown (See Appendix A) how the discrete Lévy flights are related to the fractional differential equation Eq. (26) of the superdiffusion with Riesz operator Eq. (23). For the continuous process described by Eq. (26), we derived exact analytical expressions Eqs. (36), (37), and (39) for the mean first passage time, the average total path length, and the probability of absorption by one of the boundaries, respectively. All these 
quantities are the solutions (See Appendix B) of the fractional differential equation Eq. (30) with Riesz kernel and with different right hand sides. In Section V, we have compared these analytical solutions with numerical solutions obtained for the discrete Lévy flights (See Figs. 1-3). We have shown that fractional differential formalism provides good approximation for the discrete Lévy flights in the interval with absorbing boundaries except the case of $\alpha \rightarrow 2$ and the case when the starting point is in the vicinity of an absorbing boundary. In the latter case the fractional differential formalism yields correct scaling behavior with respect to the interval size and distance to the boundary, but gives an incorrect proportionality coefficient (See Fig. 3).

In Section VI, we have investigated the behavior of the average path length as a function of the starting point and as a function of $\alpha$. We have derived asymptotic expression Eq. (45) for this quantity in the case when the starting point is located close to the absorbing boundary. We have shown that the expression for the average path length has a minimum at $\alpha \approx 1$ if the process starts in the vicinity of the absorbing boundaries (See Figs. 4,5). This result, as well as Eqs. (39) and (44), can be applied to the problem of light transmission through cloudy atmosphere 26,27].

Similar fractional integral operators [3, 13] — namely the Riesz operator $\nabla^{\alpha}$ - can be used to treat the problem of the Lévy flyer in the dimensions higher than one with randomly distributed absorbing traps. Let $L$ be a characteristic distance between neighboring traps. Then we still expect that the average number of flights before absorption scales as $L^{\alpha}$ if the process starts far away from the absorbing boundary and as $L^{\alpha / 2}$ if the process starts in the vicinity of the absorbing boundary. This result is sufficient to prove that the minimum of the average total path length traveled by the flyer before absorption is achieved at $\alpha \rightarrow 1$, if the flyer starts in the vicinity of the absorbing point.

Finally, we comment on the relevance of our findings to biological Lévy flight foraging. Our results essentially confirm that Lévy flights with $\alpha=1$ (or $\mu=2$ in notation of [36]) should theoretically provide the optimal strategy of foraging in case of sparsely and randomly located food sites, if any food site can be revisited many times [36]. The presence of the second minimum near $\alpha=2$ predicted by continuous limit approximation may indicate another possible strategy for foraging, i.e to perform Brownian walks in the region of possible appearance of prey. Break down of the continuous limit approximation in the vicinity of the absorbing boundary indicates that the results should depend on the particular details of the model.

\section{ACKNOWLEDGMENTS}

We are grateful to M. F. Shlesinger, J. Klafter, C. Tsallis, A. Marshak, A. Davis, and M. Gitterman for helpful suggestions and critical comments. We also wish to thank an anonymous referee for constructive critique that has helped to improve our manuscript. We thank NSF and CNPq for financial support. 


\section{APPENDIX A: EXISTENCE OF THE CONTINUOUS LIMIT OPERATOR}

We will show that operator $\mathcal{D}_{\alpha}$ is defined on any function $f(x)$ that has finite limits at both ends of the interval $f(0)$ and $f(L)$ and finite second derivative $f^{\prime \prime}(x)$ at any inner point $x$ of the interval $[0, L]$. According to Eqs. (6) and (22),

$$
\mathcal{D}_{\alpha} f(y)=\lim _{\ell_{0} \rightarrow 0} \ell_{0}^{-\alpha}\left\{\frac{\alpha \ell_{0}^{\alpha}}{2}\left[\int_{0}^{y-\ell_{0}} \frac{f(x) d x}{(y-x)^{\alpha+1}}+\int_{y+\ell_{0}}^{L} \frac{f(x) d x}{(x-y)^{\alpha+1}}\right]-f(y)\right\} .
$$

Making partial integration of both integrals in Eq. (A1) we get

$$
\begin{gathered}
\mathcal{D}_{\alpha} f(y)=\lim _{\ell_{0} \rightarrow 0}\left\{\ell_{0}^{-\alpha}\left[\frac{f\left(y-\ell_{0}\right)}{2}+\frac{f\left(y+\ell_{0}\right)}{2}-f(y)\right]\right. \\
\left.+\frac{1}{2}\left[-\int_{0}^{y-\ell_{0}} \frac{f^{\prime}(x) d x}{(y-x)^{\alpha}}+\int_{y+\ell_{0}}^{L} \frac{f^{\prime}(x) d x}{(x-y)^{\alpha}}\right]\right\} \\
-\frac{f(0)}{2 y^{\alpha}}-\frac{f(L)}{2(L-y)^{\alpha}} .
\end{gathered}
$$

For $\alpha<2$, the first term in Eq. (A2) goes to zero as

$$
\frac{1}{2} \ell_{0}^{2-\alpha} f^{\prime \prime}(y)
$$

The second term converges to the integral

$$
I \equiv \text { V.P. } \int_{0}^{L} \frac{\operatorname{sgn}(x-y) f^{\prime}(x) d x}{2|y-x|^{\alpha}},
$$

which exists for $\alpha<2$ if $f^{\prime}(x)$ has a derivative at $x=y$.

Subtracting Eq. (A3) from the second term in Eq. (A2), replacing $f^{\prime}(x)$ in the integrand by its Taylor expansion $f^{\prime}(x)=f^{\prime}(y)+f^{\prime \prime}(y)(x-y)+o(x-y)$, and combining it with the first term of Eq. (A2), we reproduce Eq. (25) for the correction operator $d_{\alpha}\left(\ell_{0}\right)$. This shows that the operator $\mathcal{D}_{\alpha}$ is well defined for the class of functions with existing second derivative.

\section{APPENDIX B: SONIN INVERSION FORMULA}

Equation (30) belongs to a class of generalized Abel equations. In his classical works, $\mathrm{N}$. Ya. Sonin [32] suggested a general method for solving such equations. In particular [31], an equation:

$$
\int_{0}^{L} \frac{\left[a_{1} \operatorname{sgn}(x-y)+a_{2}\right]^{\nu}}{2|x-y|^{\alpha}} \varphi(y) d y=h(x), \quad 0 \leq x \leq L
$$

has a solution: 


$$
\varphi(z)=B_{\alpha} z^{\beta-1} \frac{d}{d z} \int_{z}^{L} \frac{t^{1-\alpha}}{(t-z)^{\beta-\alpha} d t} \frac{d}{d t} \int_{0}^{t} \frac{y^{\alpha-\beta}}{(t-y)^{1-\beta}} h(y) d y
$$

where

$$
B_{\alpha}=-2 \sin (\pi \beta) \Gamma(\alpha) \Gamma^{-1}(\beta) \Gamma^{-1}(1-\beta+\alpha)\left(a_{1}+a_{2}\right)^{-\nu} \pi^{-1}
$$

and parameter $\beta$ is determined by relations

$$
\begin{gathered}
\sin (\pi(\beta-\alpha))=c_{\nu} \sin (\pi \beta), \\
c_{\nu}=\left(\frac{a_{2}-a_{1}}{a_{1}+a_{2}}\right)^{\nu} .
\end{gathered}
$$

Similar inversion formulae can be found in ref. [29].

In case of Eq. (30), $\nu=1, a_{2}=0, a_{1}=-1$. Hence, according to Eq. (BB5) $c_{\nu}=-1$. Equation (B4) has infinite number of solutions $\beta=\alpha / 2+k$, where $k$ is an integer. For $0<\alpha<2$, only two solutions with $k=0, k=1$ lead to the converging integrals in Eq. (B2):

$$
\varphi_{1}(x)=B_{\alpha} z^{\alpha / 2-1} \frac{d}{d z} \int_{z}^{L} d t t^{1-\alpha}(t-z)^{\alpha / 2} \frac{d}{d t} \int_{0}^{t} y^{\alpha / 2}(t-y)^{\alpha / 2-1} h(y) d y
$$

and

$$
\varphi_{2}(x)=-B_{\alpha} z^{\alpha / 2} \frac{d}{d z} \int_{z}^{L} d t t^{1-\alpha}(t-z)^{\alpha / 2-1} \frac{d}{d t} \int_{0}^{t} y^{\alpha / 2-1}(t-y)^{\alpha / 2} h(y) d y
$$

where

$$
B_{\alpha}=\frac{4 \sin \left(\frac{\pi \alpha}{2}\right)}{\pi \alpha \mathrm{B}\left(\frac{\alpha}{2}, \frac{\alpha}{2}\right)}
$$

Since Eq. (30) contains $f^{\prime}(x)=\varphi(x)$, one can always satisfy the first boundary condition Eq. (31) by defining $f(x)=\int_{0}^{x} \varphi(z) d z$. Adding solution (B6) for $h(x)=-1$ and solution (B7) for $h(x)=1$, one can see that the homogeneous equation with $h(x)=0$ has a nontrivial solution $\varphi_{0}=\left(L x-x^{2}\right)^{\frac{\alpha}{2}-1}$. Hence the second boundary condition Eq. (31) can be satisfied if we select $\varphi=\varphi_{1}-C \varphi_{0}$, with constant $C=L^{1-\alpha} \int_{0}^{L} \varphi(x) d x / \mathrm{B}\left(\frac{\alpha}{2}, \frac{\alpha}{2}\right)$. In case $h(x)=-1$, straightforward calculations lead to Eq. (32).

Now we will obtain the analytical solution for the average total path length before absorption in the continuous process, $S_{\alpha}(x)$. In this case, the right hand side of Eq. (30), $h(x)=s(x)$, is given by Eq. (16), and we can use its symmetry $h(x)=h(L-x)$. Thus, in order to satisfy the second boundary condition Eq. (31), we should have $\varphi(x)=-\varphi(L-x)$. To construct such a solution, we first find the solution $\varphi_{1}(x)$ for the first term in Eq. (16) $h(x)=x^{1-\alpha}$. Obviously, the function $-\varphi_{1}(L-x)$ provides the solution for the second term $h(x)=(L-x)^{1-\alpha}$. The solution for the third constant term is given by Eq. (32) with a proper coefficient. Summing up all three partial solutions and using various properties of hypergeometric functions [39], one can find the total solution presented in Eq. (37). 


\section{REFERENCES}

[1] B. D. Hughes, Random Walks and Random Environments, Volume 1: Random Walks (Clarendon Press, Oxford, 1995).

[2] M. F. Shlesinger, G. Zaslavsky, and U. Frisch, eds., Lévy Flights and Related Topics in Physics (Springer, Berlin, 1995).

[3] R. Metzler and J. Klafter, Phys. Rep. 339, 1 (2000).

[4] V. M. Zolotarev and V. M. Uchaikin, Chance and Stability: Stable Distributions and their Applications (VSP BV, Utrecht, 1999).

[5] C. Tsallis, Phys. World 10, 42 (1997)

[6] B. J. West and V. Seshadri, Physica A 113, 203 (1982).

[7] M. F. Shlesinger and J. Klafter in On Growth and Form, edited by H. E. Stanley and N. Ostrowsky (Nijhoff, Dordrecht, 1986), p. 283.

[8] B. J. West and W. Deering, Phys. Reports 246, 1 (1994).

[9] G. M. Zaslavsky, Physics of Chaos in Hamiltonian System (Imperial College Press, London, 1998)

[10] R. Kutner, Physica A 264, 84 (1999), R. Kutner, A. Pekalski, K. Sznajd-Weron (Eds.), Anomalous Diffusion: from Basics to Applications (Springer-Verlag, Berlin 1999).

[11] G. Samorodnitsky and M. S. Taqqu, Stable Non-Gaussian Random Processes (Chapman \& Hall, New York, 1994)

[12] G. M. Zaslavsky, Physica D 76, 110 (1994).

[13] H. C. Fogedby, Phys. Rev. E. 58, 1690 (1998).

[14] S. Jespersen, R. Metzler, and H. C. Fogedby, Phys. Rev. E. 59, 2736 (1999).

[15] A. I. Saichev and G. M. Zaslavsky, Chaos 7, 753 (1997).

[16] M. Gitterman, Phys. Rev. E 62, 6065 (2000).

[17] W. R. Schneider and W. Wyss, J. Math, Phys. 30, 134 (1989).

[18] E. Barkai and R.J. Sibley, J. Phys. Chem. B 104, 3866 (2000).

[19] R. Metzler, E. Barkai, and J. Klafter, Phys. Rev. Lett. 823563 (1999).

[20] R. Metzler and J. Klafter, Physica A 278, 107 (2000).

[21] R. Metzler and J. Klafter, Europhys. Lett. 51, 492 (2000).

[22] G. Rangarajan and M. Ding, Phys. Rev. E. 62, 120 (2000).

[23] E. Barkai, Phys. Rev. E. 63, 046118 (2001).

[24] I. Podlubny, Fractional Differential Equations (Academic Press, London, 1999).

[25] P. M. Drysdale and P. A. Robinson, Phys. Rev. E 58, 5382 (1998).

[26] A. Davis and A. Marshak in Fractal Frontiers edited by M. M. Novak and T. G. Dewey (World Sci. River Edge, N.J.,1997) p. 63.

[27] K. Pfeilsticker, J. Geophys. Res. D 1044101 (1999).

[28] V. I. Smirnov, A course of higher mathematics, vol. 4: Integral equations (Pergamon Press, Oxford, 1964).

[29] S. G. Samko, A. A. Kilbas, and O. I. Maritchev, Integrals and Derivatives of the Fractional Order and Some of Their Applications (Nauka i Tekhnika, Minsk, 1987) [in Russian]; S. G. Samko, A. A. Kilbas, and O. I. Maritchev, Fractional Integrals and Derivatives (Gordon and Breach, Newark 1993).

[30] N. Kh. Arutyunyan, Prikl. Mat. Mekh. 23, 901 (1959) [in Russian].

[31] G. Ya. Popov, Stress Construction near Punches, Cuts, Thin Inclusions, and Supporters (Nauka, Moskow, 1982) [in Russian]. 
[32] N. Ya. Sonin, Studies on cylinder functions and special polynomials (Gostekhizdat, Moscow, 1954) [in Russian].

[33] M. Levandowsky, J. Klafter, and B. S. White, Bull. Mar. Sci. 43, 758 (1988).

[34] B. J. Cole, Anim. Behav. 50, 1317 (1995).

[35] G. M. Viswanathan, V. Afanasyev, S. V. Buldyrev, E. J. Murphy, P. A. Prince and H. E. Stanley, Nature 381, 413 (1996).

[36] G. M. Viswanathan, S. Buldyrev, S. Havlin, M. G. E. da Luz, E. P. Raposo and H. E. Stanley, Nature 401, 911 (1999).

[37] G. M. Viswanathan, V. Afanasyev, S. V. Buldyrev, S. Havlin, M. G. E. da Luz, E. P. Raposo, and H. E. Stanley, Physica A 282, 1 (2000).

[38] A. Upadhyaya, J.-P. Rieu, J. A. Glazier, and Y. Sawada, Physica A 293, 549 (2001).

[39] M. Abramowitz and I. A. Stegun, Handbook of Mathematical Functions (Dover Publications Inc., New York, 1965); V. I. Smirnov, A course of higher mathematics, vol. 3, part 2: Complex variables, special functions (Pergamon Press, Oxford, 1964).

[40] H. M. Kogan J. Uspekhi Mat. Nauk, 19 no 4, 228 (1964). [In Russian]

[41] S. V. Buldyrev and M. Gitterman (unpublished).

[42] Note that one can define $\mathcal{D}_{\alpha} \equiv \lim _{\ell_{0} \rightarrow 0} \ell_{0}^{-2}\left[\mathcal{L}_{\alpha}\left(\ell_{0}\right)-\mathcal{I}\right]=\frac{\alpha}{2(\alpha-2)} \frac{d^{2}}{d x^{2}}$ for $\alpha>2$, and $\mathcal{D}_{\alpha} \equiv \lim _{\ell_{0} \rightarrow 0} \ell_{0}^{-2}\left(-\ln \ell_{0}\right)^{-1}\left[\mathcal{L}_{\alpha}\left(\ell_{0}\right)-\mathcal{I}\right]=\frac{d^{2}}{d x^{2}}$ for $\alpha=2$. 


\section{FIGURES}

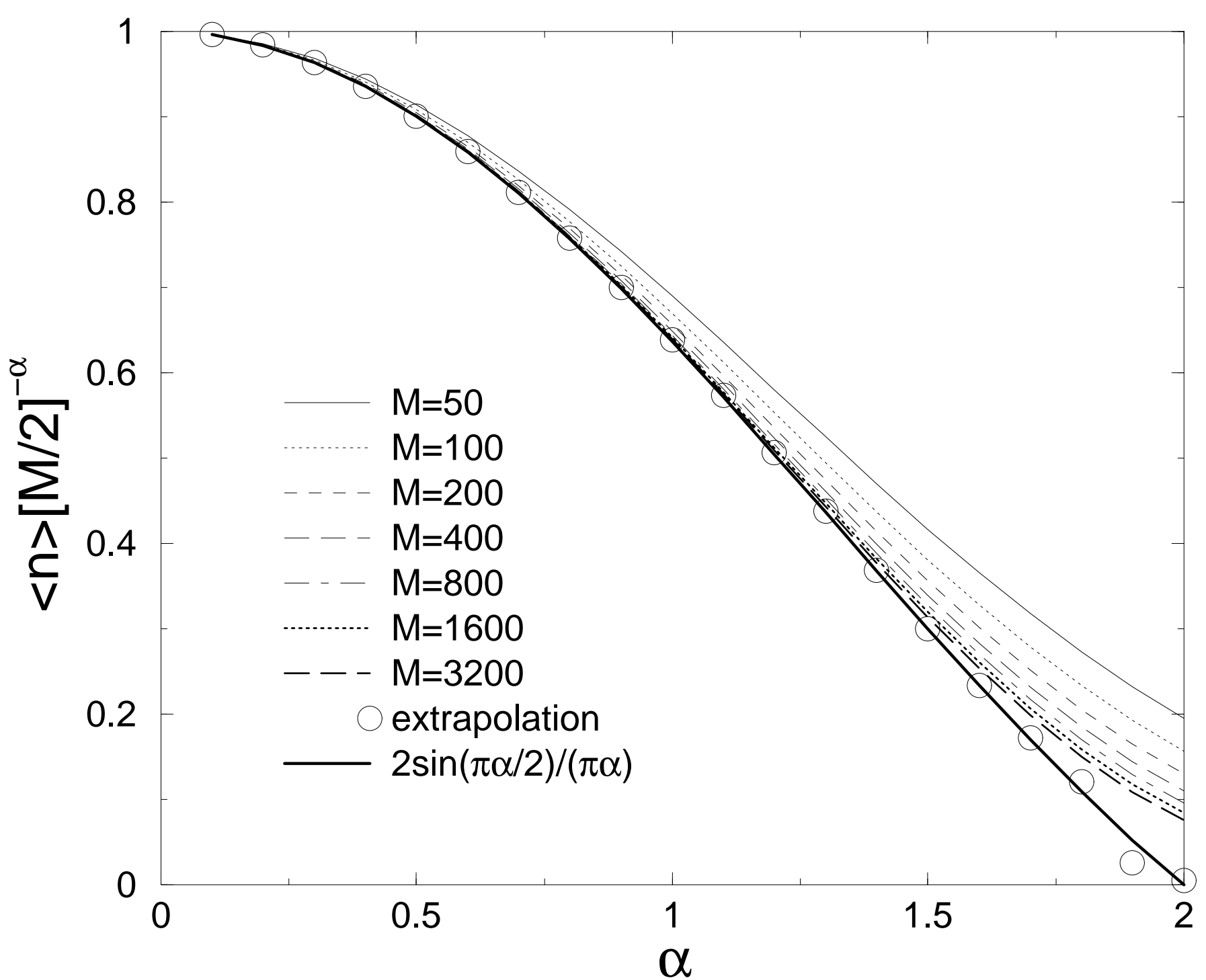

FIG. 1. The behavior of the scaled average number of flights $\langle n\rangle(M / 2)^{-\alpha}$ versus $\alpha$ for increasing values of $M=50,100,200,400,800,1600,3200$ in the case $x_{0}=L / 2$ in comparison with the continuous limit prediction of Eq. (36), $2 \sin (\pi \alpha / 2) /(\pi \alpha)$, shown as a bold line. We see good convergence to the predicted function except for the values of $\alpha \approx 2$. We extrapolate the values $\langle n\rangle(M / 2)^{-\alpha}$ for $M \rightarrow \infty$ (circles) using their polynomial fits with respect to $M^{-1}$ for $\alpha \leq 1$, or with respect to $M^{2-\alpha}$ for $1<\alpha<2$, or with respect to $(\ln M)^{-1}$ for $\alpha=2$. 


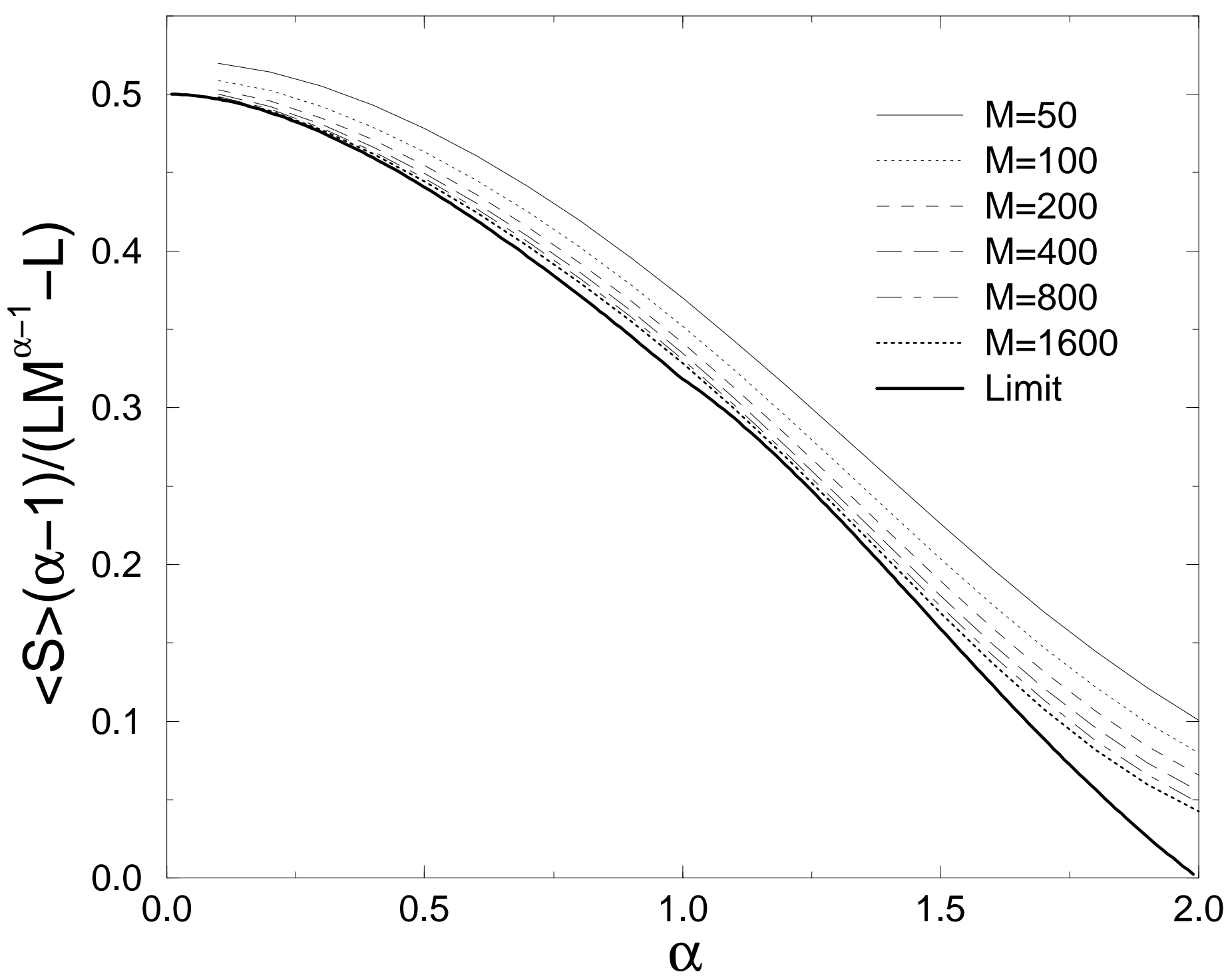

FIG. 2. The behavior of the scaled average total path length $\langle S\rangle(\alpha-1) /\left(L M^{\alpha-1}-L\right)$ versus $\alpha$ for increasing values of $M=50,100,200,400,800,1600$ in the case $x_{0}=L / 2$ in comparison with the continuous limit prediction of Eq. (37) in the limit $M \rightarrow \infty$ shown as a bold line. In this limit, continuous approximation follows first term of Eq. (37) for $\alpha<1$ and the second term $\sin (\pi \alpha / 2) / \pi$ for $\alpha \geq 1$. We see good convergence to the predicted function except for the values of $\alpha \approx 2$. 


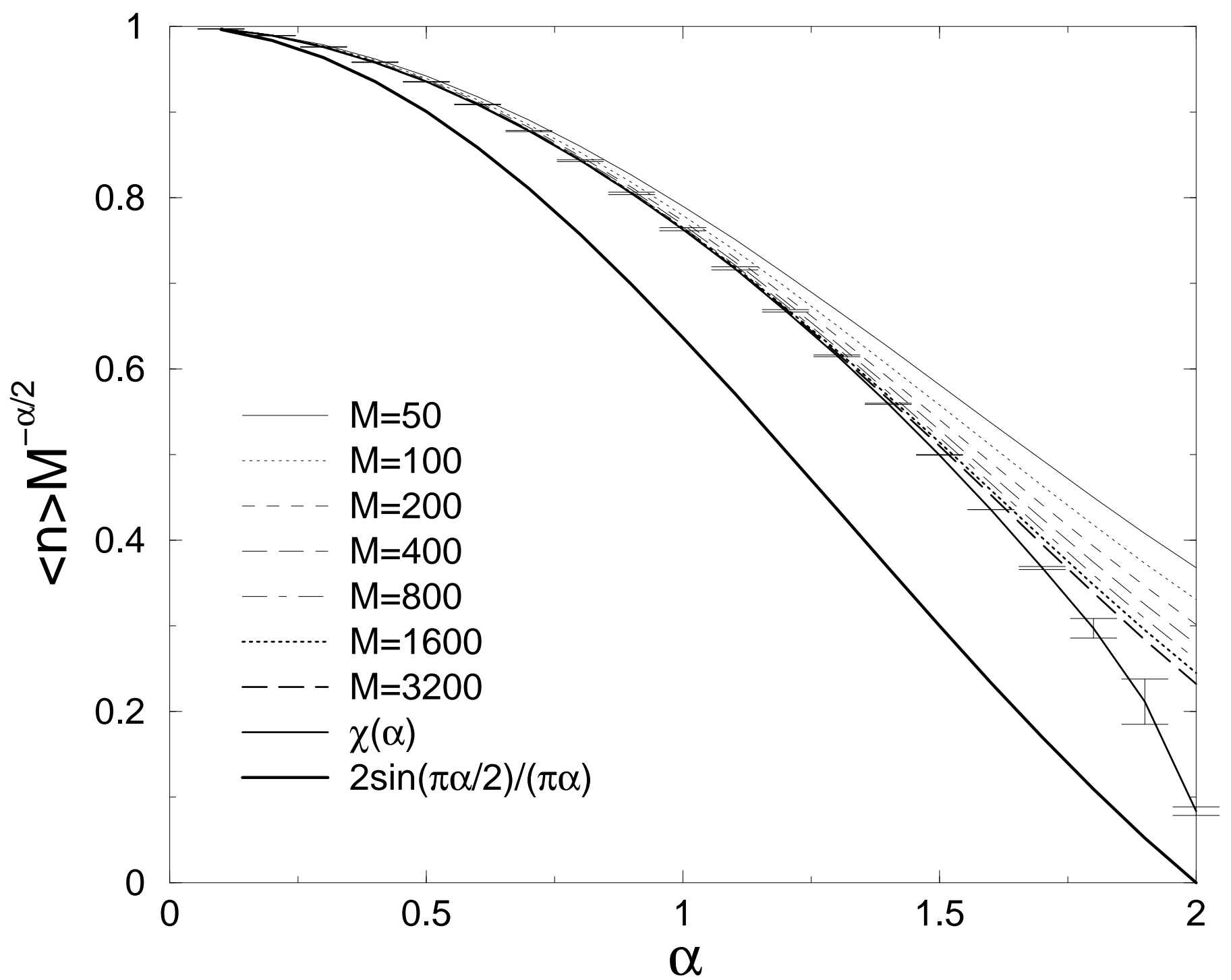

FIG. 3. The behavior of the scaled average number of flights $\langle n\rangle(M)^{-\alpha / 2}$ versus $\alpha$ for increasing values of $M=50,100,200,400,800,1600,3200$ in the case $x_{0}=\ell_{0}$ in comparison with the continuous limit prediction of Eq. (36) $2 \sin (\pi \alpha / 2) /(\pi \alpha)$, shown as a bold line. Although the values are close to the continuous limit predictions, they converge to a different function $\chi(\alpha)$ as $M \rightarrow \infty$. To obtain $\chi(\alpha)$, we extrapolate the values $\langle n\rangle(M / 2)^{-\alpha}$ for $M \rightarrow \infty$ using the same procedure as in Fig. 1. We assume that the error bars are equal the discrepancies between the extrapolation and the continuous limit in Fig. 1. 


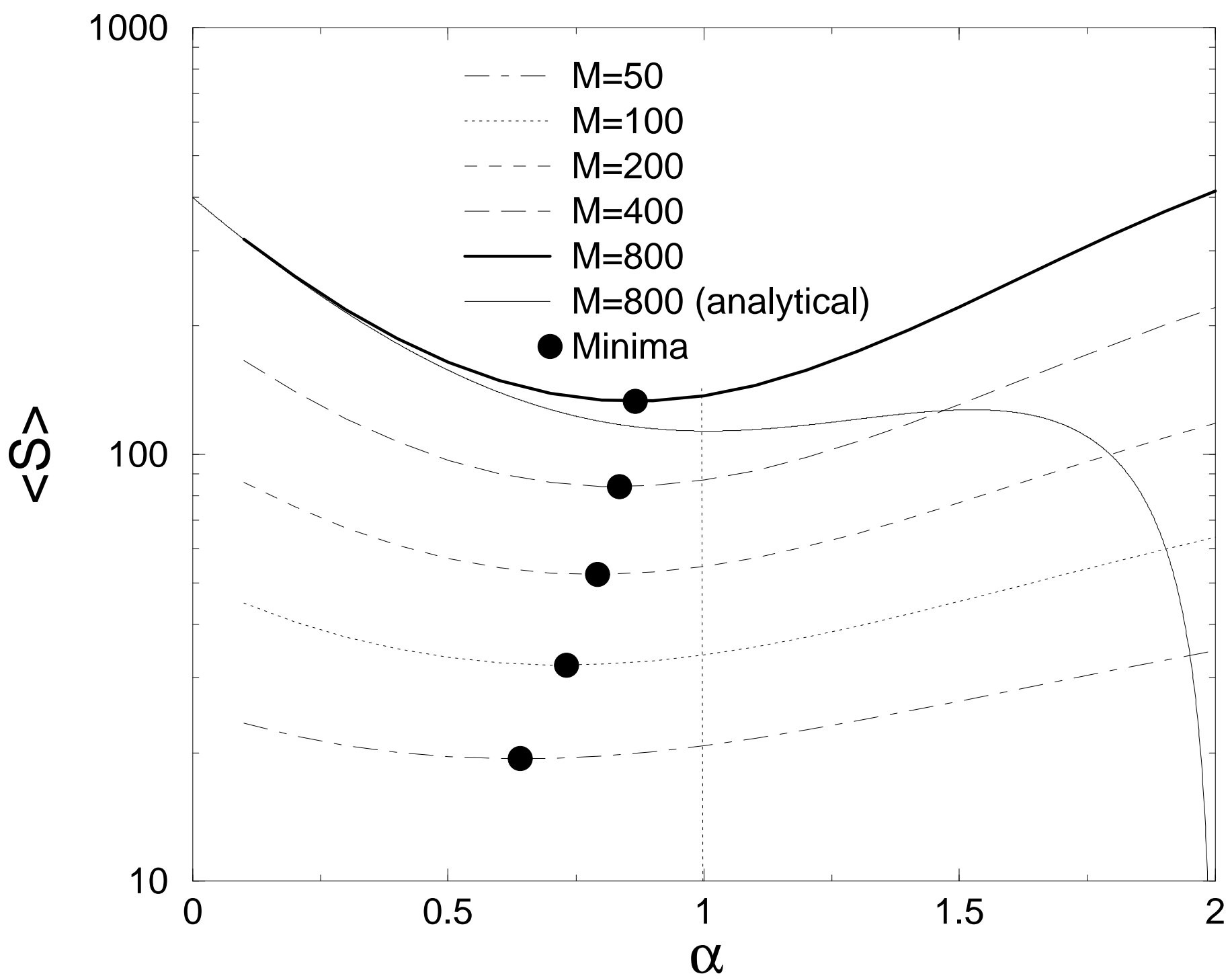

FIG. 4. Semi-logarithmic plot of numerical solutions of $\langle S\rangle$ versus $\alpha$ for the case $x_{0}=\ell_{0}=1$ and various values of $M=50,100,200,400,800$. Circles indicate the positions of the minima $\alpha_{\min }(M)$ which shift towards the vertical line $\alpha=1$, as $M$ increases. In addition, we show the analytical continuous limit approximation $S_{\alpha}\left(\ell_{0}\right)$ given by Eq. (46) for $M=800$. 


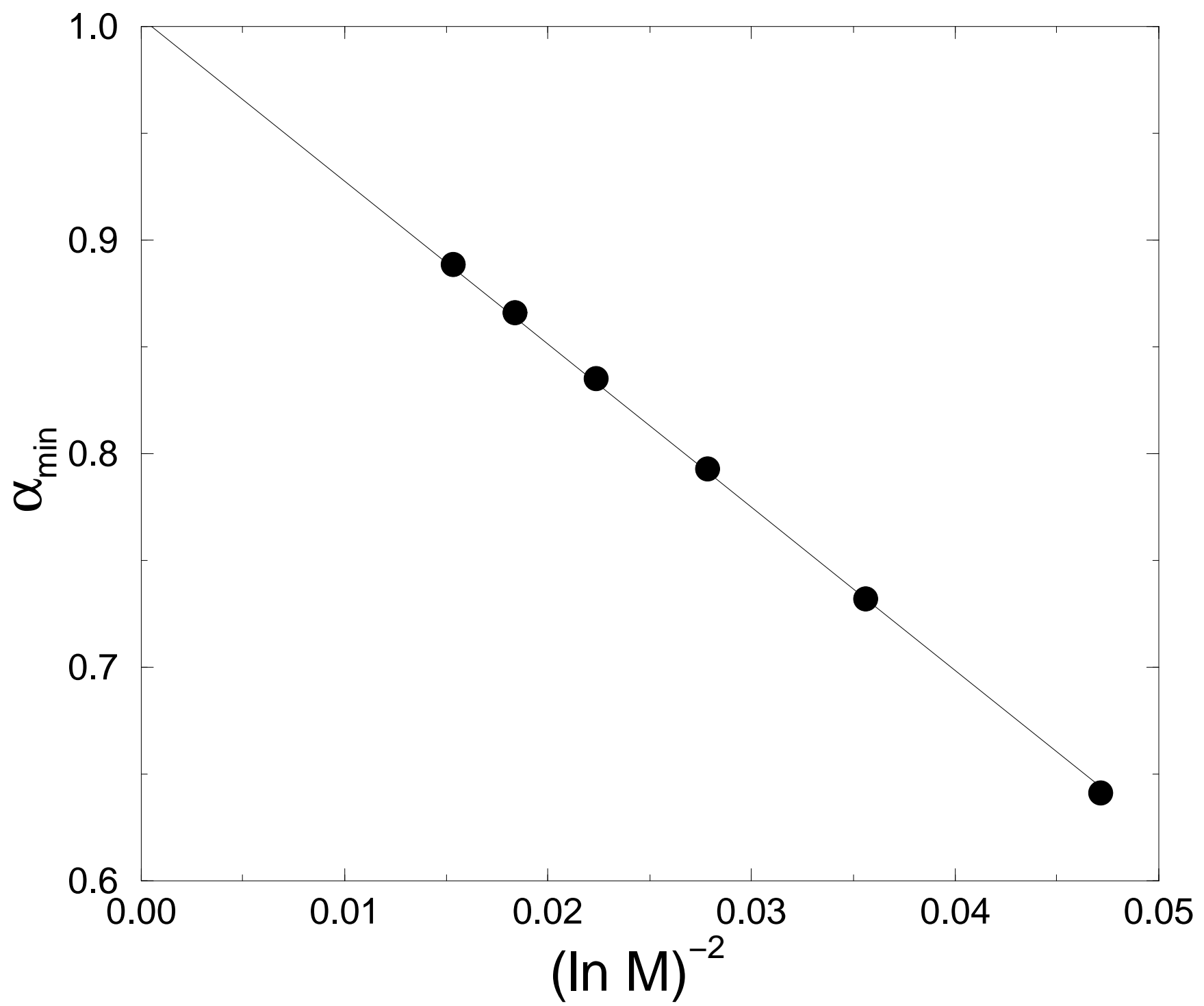

FIG. 5. The values of $\alpha_{\min }(M)$, determined in Fig. 4 as a function of $(\ln M)^{-2}$. The line shows linear least square fit, obtained by including a hypothetical limiting value $\alpha_{\min }(\infty)=1$. 